\title{
Choice degrees in decision-making: A comparison between intuitionistic and fuzzy preference relations approaches
}

\author{
Francisco Chiclana*, Raquel Ureña ${ }^{\dagger}$, Enrique Herrera-Viedma $^{\dagger}$ \\ ${ }^{*}$ Centre for Computational Intelligence (CCI), Faculty of Technology \\ De Montfort University, Leicester, UK \\ E-mail: chiclana@dmu.ac.uk \\ $\dagger$ Department of Computer Science and Artificial Intelligence \\ University of Granada, Granada, Spain \\ Email: \{raquel,viedma\}@ decsai.ugr.es
}

\begin{abstract}
Preference modelling based on Atanassov's intuitionistic fuzzy sets are gaining increasing relevance in the field of group decision making as they provide experts with a flexible and simple tool to express their preferences on a set of alternative options, while allowing, at the same time, to accommodate experts' preference uncertainty, which is inherent to all decision making processes. A key issue within this framework is the provision of efficient methods to rank alternatives, from best to worse, taking into account the peculiarities that this type of preference representation format presents. In this contribution we analyse the relationships between the main method proposed and used by researchers to rank alternatives using intuitionistic fuzzy sets, the score degree function, and the well known choice degree based on Orlovsky's non-dominance concept for the case when the preferences are expressed by means of fuzzy preference relations. This relationship study will provide the necessary theoretical results to support the implementation of Orlovsky's non-dominance concept to define the fuzzy quantifier guided non-dominance choice degree for intuitionistic fuzzy preference relations.
\end{abstract}

\section{INTRODUCTION}

Intuitionistic fuzzy sets (IFSs), which are characterised by three functions expressing degrees of membership, nonmembership, and hesitation (or indeterminacy) have received increasing attention since they were proposed by Atanassov [2]. This is mainly due to their flexibility in handling vagueness and uncertainty. For this reason, intuitionistic fuzzy set theory has been extensively used in many research areas, such as virtual medical diagnosis [12], pattern recognition [23], clustering analysis [28] and decision making [21], [24]-[26].

In any decision making process, after the opinions of the experts have been fused using an appropriate aggregation operator, the aggregated Intuitionistic Fuzzy Numbers (IFNs) are compared to produce a final ranking of the alternatives. However, according to Yager [30], the comparison of Fuzzy Numbers (FNs) is a problem that has been extensively studied but no unique best approach has been found yet. Most of the time, the choosing of the comparison method to use is a matter of preference or might be context dependent. Indeed a widely used ranking approach, which was originally proposed by Zadeh in [31], translates the FNs into a representative crisp value, and performs the comparison on them [19], [30]. Recall that a FN is particular type of IFN. Thus, the same conclusion applies to the latter. Consequently, an important research topic regards the development of methodologies to make possible the comparison of criterion values that are expressed by IFSs in order to produce a ranking of them. Among the existing comparison approaches of IFNs, it worth highlighting the predominantly use of methods based on the score and accuracy degree functions. A comprehensive comparative analysis of existing score degree and accuracy degree functions has been reported in [7].

An alternative ranking methodology is the one based on the concept of non-dominance degree [14] of one alternative over the fuzzy majority of the rest of the alternatives, which extends the original non-dominance degree of preference introduced by Orlovsky's in [18]. This methodology is well used and developed in a framework where opinions are represented using fuzzy preference relations and/or fuzzy linguistic preference relations [1], [15].

Although a substantial number of studies have examined decision approaches using score functions in the context of intuitionistic fuzzy preference relations and dominance and non-dominance degrees for fuzzy preference relations, the issue of comparing the solutions yielded by means of both approaches has not been explored yet. Therefore, the main objective of this contribution is to analyse the relationship between both types of ranking approaches when working with intuitionistic fuzzy preference relations. To carry out this study, the mathematical equivalence between the set of reciprocal intuitionistic fuzzy preference relations and the set of asymmetric fuzzy preference relations pointed out by the authors in [22] will be exploited, as well as the score fuzzy preference relation associated to an intuitionistic fuzzy preference relation proposed in [24].

The rest of the paper is set out as follows: Section II presents the main mathematical frameworks for representing preferences and the basics concepts needed throughout the 
rest of the paper. The one-to-one correspondence between the set of intuitionistic fuzzy preference relations and the set of asymmetric fuzzy preference relations is revisited as well in this section. Section III briefly reviews the extensive research on ranking functions by means of score and accuracy degrees and also by means of fuzzy quantifier guided dominance and fuzzy quantifier non-dominance selection approaches. This section also revisits the reciprocal score fuzzy preference relation associated to an intuitionistic fuzzy preference relation. The comparative analysis of the ranking approaches for intuitionistic fuzzy preference relations and fuzzy preference relations is provided in Section IV. This study will provide the mathematical foundation to introduce Orlovsky's nondominance concept to define the fuzzy quantifier guided nondominance choice degree for intuitionistic fuzzy preference relations. Finally, Section V draws conclusions and presents some future work.

\section{Preference Relations in Decision MaKing}

In group decision making problems, once the set of feasible alternatives $(X)$ is identified, experts are called to express their opinions or preferences on such set. Different preference elicitation methods were compared in [17], where it was concluded that pairwise comparison methods are more accurate than nonpairwise methods because they allow the expert to focus on two alternatives at a time. A comparison of two alternatives by an expert can lead to the preference of one alternative to the other or to a state of indifference between them. Obviously, there is the possibility of an expert being unable to compare them. Two main mathematical models based on the concept of preference relation can be used in this context. In the first one, a preference relation is defined for each one of the above three possible preference states mentioned above (preference, indifference, incomparability) [11], which is usually referred to as a preference structure on the set of alternatives [20]. The second one integrates the three possible preference states into a single preference relation [3]. In this paper, we focus on the second one as per the following definition:

Definition 1 (Preference Relation). A preference relation $P$ on a set $X$ is a binary relation $\mu_{P}: X \times X \rightarrow D$, where $D$ is the domain of representation of preference degrees provided by the decision maker.

A preference relation $P$ may be conveniently represented by a matrix $P=\left(p_{i j}\right)$ of dimension $\# X$, with $p_{i j}=\mu_{P}\left(x_{i}, x_{j}\right)$ being interpreted as the degree or intensity of preference of alternative $x_{i}$ over $x_{j}$. The elements of $P$ can be of a numeric or linguistic nature, i.e., could represent numeric or linguistic preferences, respectively. The main types of numeric preference relations used in decision making are: crisp preference relations, additive preference relations, multiplicative preference relations, interval-valued preference relations and intuitionistic preference relations. A comprehensive survey of them have been reported on [27], which the reader is encouraged to consult for further particulars. In this contribution, the focus is on fuzzy preference relations and intuitionistic fuzzy preference relations.

\section{A. Fuzzy Set and Fuzzy Preference Relation}

Definition 2 (Fuzzy Set). Let $U$ be a universal set defined in a specific problem, with a generic element denoted by $x$. A fuzzy set $X$ in $U$ is a set of ordered pairs:

$$
X=\left\{\left(x, \mu_{X}(x)\right) \mid x \in U\right\}
$$

where $\mu_{X}: U \rightarrow[0,1]$ is called the membership function of $A$ and $\mu_{X}(x)$ represents the degree of membership of the element $x$ in $X$.

The degree of non-membership of the element $x$ in $X$ is here defined as $v_{X}(x)=1-\mu_{X}(x)$. Thus, $\mu_{X}(x)+v_{X}(x)=1$.

Definition 3 (Fuzzy Preference Relation). A fuzzy preference relation $R=\left(r_{i j}\right)$ on a finite set of alternatives $X$ is a fuzzy relation in $X \times X$ that is characterised by a membership function $\mu_{R}: X \times X \longrightarrow[0,1]$ with the following interpretation:

- $r_{i j}=1$ indicates the maximum degree of preference for $x_{i}$ over $x_{j}$

- $\left.r_{i j} \in\right] 0.5,1\left[\right.$ indicates a definite preference for $x_{i}$ over $x_{j}$ - $r_{i j}=1 / 2$ indicates indifference between $x_{i}$ and $x_{j}$

When

$$
r_{i j}+r_{j i}=1 \forall i, j \in\{1, \ldots, n\}
$$

is imposed the fuzzy preference relation is called reciprocal.

\section{B. Intuitionistic Fuzzy Set and Intuitionistic Fuzzy Preference} Relation

The concept of an Intuitionistic Fuzzy Set (IFS) was introduced by Atanassov in [2]:

Definition 4 (Intuitionistic Fuzzy Set). An intuitionistic fuzzy set $X$ over a universe of discourse $U$ is given by

$$
X=\left\{\left(x,\left\langle\mu_{X}(x), v_{X}(x)\right\rangle\right) \mid x \in U\right\}
$$

where $\mu_{X}: U \rightarrow[0,1]$, and $v_{X}: U \rightarrow[0,1]$ verify

$$
0 \leq \mu_{X}(x)+v_{X}(x) \leq 1 \quad \forall x \in U
$$

$\mu_{X}(x)$ and $v_{X}(x)$ represent the degree of membership and degree of non-membership of $x$ in $X$, respectively.

An intuitionistic fuzzy set becomes a fuzzy set when $\mu_{X}(x)=1-v_{X}(x) \forall x \in U$. However, when there exists at least one value $x \in U$ such that $\mu_{X}(x)<1-v_{X}(x)$, an extra parameter has to be taken into account when working with intuitionistic fuzzy sets: the hesitancy degree, $\tau_{X}(x)=1-$ $\mu_{X}(x)-v_{X}(x)$, that represents the amount of lacking information in determining the membership of $x$ to $X$. If the hesitation degree is zero, the reciprocal relationship between membership and non-membership makes the latter one unnecessary in the formulation as it can be derived from the former.

Szmidt and Kacprzyk in [21] defined the intuitionistic fuzzy preference relation as a generalisation of the concept of fuzzy preference relation. 
Definition 5 (Intuitionistic Fuzzy Preference Relation). An intuitionistic fuzzy preference relation $B$ on a finite set of alternatives $X=\left\{x_{1}, \ldots, x_{n}\right\}$ is characterised by a membership function $\mu_{B}: X \times X \rightarrow[0,1]$ and a non-membership function $v_{B}: X \times X \rightarrow[0,1]$ such that

$$
0 \leq \mu_{B}\left(x_{i}, x_{j}\right)+v_{B}\left(x_{i}, x_{j}\right) \leq 1 \quad \forall\left(x_{i}, x_{j}\right) \in X \times X .
$$

with $\mu_{B}\left(x_{i}, x_{j}\right)=\mu_{i j}$ interpreted as the certainty degree up to which $x_{i}$ is preferred to $x_{j}$; and $v_{B}\left(x_{i}, x_{j}\right)=v_{i j}$ interpreted as the certainty degree up to which $x_{i}$ is non-preferred to $x_{j}$.

An intuitionistic fuzzy preference relation can also be represented by a matrix $B=\left(b_{i j}\right)$ with $b_{i j}=\left\langle\mu_{i j}, v_{i j}\right\rangle \forall i, j=$ $1,2, \ldots, n$, and it is referred to as reciprocal when the following additional conditions are impossed:

- $\mu_{i i}=v_{i i}=0.5 \forall i \in\{1, \ldots, n\}$.

- $\mu_{j i}=v_{i j} \forall i, j \in\{1, \ldots, n\}$.

Notice that when the hesitancy degree function is the null function in a reciprocal intuitionistic fuzzy preference relation, $B=\left(b_{i j}\right)=\left(\left\langle\mu_{i j}, v_{i j}\right\rangle\right)$, then it is $\mu_{i j}+v_{i j}=1 \forall i, j$, and $B$ is mathematically equivalent to the reciprocal fuzzy preference relation $R=\left(r_{i j}\right)=\left(\mu_{i j}\right)$.

\section{Reciprocal Intuitionistic Fuzzy Preference Relations and} Asymmetric Fuzzy Preference Relations

Let us denote with $\mathscr{B}$ the set of reciprocal intuitionistic fuzzy preference relations:

$$
\begin{aligned}
\mathscr{B}=\left\{B=\left(b_{i j}\right) \mid \forall i j: \quad b_{i j}\right. & =<\mu_{i j}, v_{i j}>, \mu_{i j}, v_{i j} \in[0,1], \\
\mu_{i i}=v_{i i} & \left.=0.5 \mu_{i j}=v_{j i}, 0 \leq \mu_{i j}+v_{i j} \leq 1\right\}
\end{aligned}
$$

and with $\mathscr{R}$ the set of fuzzy preference relations

$$
\mathscr{R}=\left\{R=\left(r_{i j}\right) \mid \forall i j: r_{i j} \in[0,1]\right\}
$$

Let us define the following mapping $f: \mathscr{B} \longrightarrow \mathscr{R}$

$$
f(B)=\left(f\left(b_{i j}\right)\right)=\left(\mu_{i j}\right)=\left(r_{i j}\right)=R .
$$

We have:

- Function $f$ is well defined, i.e. given $B \in \mathscr{B}$ it is true that $f(B) \in \mathscr{R}$.

- Function $f$ is an injection. Indeed, let $B_{1}$ and $B_{2}$ two reciprocal intuitionistic fuzzy preference relations such that $f\left(B_{1}\right)=f\left(B_{2}\right)$. Then we have that

$$
f\left(r_{i j}^{1}\right)=f\left(r_{i j}^{2}\right) \Leftrightarrow \mu_{i j}^{1}=\mu_{i j}^{2} \forall i, j .
$$

Because $\mu_{i j}^{1}=v_{j i}^{1}$ and $\mu_{i j}^{2}=v_{j i}^{2}$ then it is obvious that

$$
v_{i j}^{1}=v_{i j}^{2} \forall i, j .
$$

Therefore we have that

$$
b_{i j}^{1}=<\mu_{i j}^{1}, v_{i j}^{1}>=<\mu_{i j}^{2}, v_{i j}^{2}>=b_{i j}^{2} \forall i, j .
$$

Consequently, it is concluded that

$$
B_{1}=B_{2} \text {. }
$$

- Function $f$ is not a surjection as not all fuzzy preference relations $R \in \mathscr{R}$ verify $0 \leq r_{i j}+r_{j i} \leq 1$. Thus the range of function function $f$ is the set of asymmetric fuzzy preference relations.

Summarising:

There exists a one-to-one correspondence between the set of reciprocal intuitionistic fuzzy preference relations and the set of asymmetric fuzzy preference relations.

This result can be exploited to define concepts for an intuitionistic fuzzy preference relation via the equivalent known ones of the associated asymmetric fuzzy preference relation. In particular, a methodology to derive a priority vector for an intuitionistic fuzzy preference relation via its corresponding fuzzy preference relation based on the concept of non-dominance degree and to tackle the issue of incomplete information in intuitionistic fuzzy preference relations in the framework of group decision making was presented in [22].

\section{RANKING APPROACHES}

Ranking fuzzy numbers is often a necessary step in many mathematical models specially in decision making under uncertainty where the best alternative from all the available ones needs to obtained. There are various approaches in the literature to rank fuzzy numbers. As it was already mentioned, a widely used approach to rank fuzzy numbers consists in converting them into a representative crisp value, and performs the comparison on them, a methodology originally proposed by Zadeh in [31]. This approach has been proposed and used in the selection process of decision making problems under uncertainty where ranking of fuzzy or intuitionistic fuzzy sets is a must to arrive at a decision [30]. Recently, a study by Brunelli and Mezei [4] that compares different ranking methods for fuzzy numbers concludes that it is impossible to give a final answer to the question on what ranking method is the best. Most of the time choosing a method rather than another is a matter of preference or is context dependent.' Two defuzzification methods widely used in fuzzy set theory are: the centre of area method (COA) and the mean of maximum method (MOM). The first one computes the centre of mass of the membership function of the fuzzy set (the centroid), whereas the second one computes the mid-point of the 1-level set of the fuzzy set. In a fuzzy linguistic framework, the COA method maintains the usually assumed underlying semantic ranking relation within the set of fuzzy linguistic labels used to assess preferences, i.e. given two fuzzy linguistic labels $l_{i}, l_{j}$ such that $\operatorname{COA}\left(l_{i}\right)<C O A\left(l_{j}\right)$ then it is $l_{i}<l_{j}$, and therefore it is frequently used in such context. It is worth mentioning that Brunelli and Mezei's correlation study, and their centrality analysis associated to the corresponding correlation network representation, shows the centre of area method as one of the highest central defuzzification methods. For a trapezoidal 
fuzzy number $\widetilde{A}=\left(t_{1}, t_{2}, t_{3}, t_{4}\right)$ with membership function

$$
\mu_{\widetilde{A}}(u)= \begin{cases}\frac{u-t_{1}}{t_{2}-t_{1}} & \text { if } t_{1}<u<t_{2} ; \\ \frac{t_{4}-u}{t_{4}-t_{3}} & \text { if } t_{2} \leq u \leq t_{3} \\ 0 & \text { otherwise. }\end{cases}
$$

Definition 7 (Hong and Choi's accuracy functions [16]). Given an IFS A over X, with IFNs $A(x)=\left\{\left[\mu_{A}(x), \mu_{A}(x)+\right.\right.$ $\left.\left.\tau_{A}(x)\right] \mid x \in X\right\}$, the accuracy degree function of $A$ is represented by

$$
\begin{gathered}
A_{C T}(A): X \rightarrow[0,1] \\
A_{H C}(A)(x)=\mu_{A}(x)+v_{A}(x)
\end{gathered}
$$

Notice that $S_{C T}(\alpha) \in[-1,1]$ while $A_{H C}(\alpha) \in[0,1]$, and that both functions are related as follows:

$$
A_{H C}(A)(x)=S_{C T}(A)(x)+2 \cdot v_{A}(x)
$$

Under the assumed property of internal symmetry of the fuzzy linguistic labels, i.e. for isosceles trapezoids with equal left and right side lengths, it is

$$
C O A=\frac{t_{4}-t_{1}}{2}=\frac{t_{3}-t_{2}}{2}=M O M .
$$

Later in the paper it is shown the apparent resemble between this last expression of $C O A(\widetilde{A})$ and the score function for an IFN.

In this section, we focus on the ranking methods more extensively used in group decision making for the case when the experts preferences have been expressed by means of fuzzy preference relations and/or intuitionistic fuzzy preference relations.

\section{A. Ranking Approaches for Intuitionistic Fuzzy Sets}

When working with IFSs there are two evaluation dimensions: one for a positive outcome expectation (membership degree) and one for a negative outcome expectation (non membership degree). The score function can be viewed as the net predisposition to signify the aggregated effect of positive and negative evaluations.

The score function was first introduced and used by Chen and Tan [6] to measure a vague value for multi-criteria fuzzy decision making. In this case, given a Vague Set (VS) [13], the score function was defined as the degree of membership minus the degree of non-membership for such type of set. Notice that since Bustince and Burillo proved in [5] that VSs are IFSs, score functions can also be applied to measure the degree of suitability of each alternative with respect to a set of criteria presented in terms of intuitionistic fuzzy values.

Definition 6 (Chen and Tan's IFS score function [6]). Given an IFS A over $X$, with IFNs $A(x)=\left\{\left[\mu_{A}(x), \mu_{A}(x)+\tau_{A}(x)\right] \mid x \in\right.$ $X\}$, the score degree function of $A$ is represented by

$$
\begin{gathered}
S_{C T}(A): X \rightarrow[0,1] \\
S_{C T}(A)(x)=\mu_{A}(x)-v_{A}(x)
\end{gathered}
$$

Later, Hong and Choi [16] introduced the accuracy function as the sum of the membership function and the nonmembership function of a vague number, pointing out that the relationship between the score function and the accuracy function is similar to the one between the mean and the variance in statistics.
Thus, the following result is proved:

Proposition 1. Given an IFN $\alpha=\langle\mu, v\rangle$, the following inequality holds:

$$
S_{C T}(A) \leq A_{H C}(A)
$$

Notice that when hesitancy is the null function, accuracy does not play any role because it is always equal to 1 .

Due to the fact that the score function is increasing with respect to the degree of membership and decreasing with respect to the degree of non-membership, from the point of view of experts' preferences about alternatives, the score value can be interpreted as the degree of suitability/satisfaction of $x$ with the decision maker's requirement. Thus, given a set of alternatives $X=\left\{x_{1}, \ldots, x_{n}\right\}$ evaluated by a decision maker against a particular criterion using an IFS $A$ over $X$, the score evaluations associated to the alternatives $\left\{S_{C T}(A)\left(x_{i}\right) ; i=\right.$ $1, \ldots, n\}$ can be used to rank them using the following ordering criterion rule:

$$
x_{i} \prec x_{j} \Leftrightarrow S_{C T}(A)\left(x_{i}\right)<S_{C T}(A)\left(x_{j}\right) .
$$

$\mathrm{Wu}$ and Chiclana proposed in [24] a new score function that allows the derivation of a fuzzy preference relation (FPR) from an intuitionistic fuzzy preference relation (IFPR).

Definition 8 (Wu and Chiclana's IFS score function). Given an IFS A over $X$, with IFNs $A(x)=\left\{\left[\mu_{A}(x), \mu_{A}(x)+\tau_{A}(x)\right] \mid x \in\right.$ $X\}$, the following score function can be computed

$$
\begin{gathered}
S_{W C}(A): X \rightarrow[0,1] \\
S_{W C}(A)(x)=\mu_{A}(x)+\frac{\tau_{A}(x)}{2}
\end{gathered}
$$

This new score function can be re-written as follows:

$$
S_{W C}(A)(x)=\frac{\mu_{A}(x)-v_{A}(x)+1}{2} .
$$

This means that $S_{W C}(A)$ and $S_{C T}(A)$ are ordering mathematically equivalent and so they will lead to the same ordering of alternatives when the previous ordering rule (5) is applied. 


\section{B. Score FPR Associated to an IFPR}

$\mathrm{Wu}$ and Chiclana present in [24] a method to derive a FPR from an IFPR $B=\left(b_{i j}\right)$ via the application of the score function $S_{W C}(A)$ (6), which it is called the score FPR (SFPR).

Proposition 2 (Score FPR (SFPR)). Let $B=\left(b_{i j}\right)$ be an IFPR. Then $P=\left(p_{i j}\right)$ where

$$
p_{i j}=S_{W C}\left(b_{i j}\right)
$$

is a reciprocal FPR. $P$ is called the score FPR (SFPR) associated to the IFPR B.

Proof. Indeed, it is

$p_{i j}+p_{j i}=S_{W C}\left(b_{i j}\right)+S_{W C}\left(b_{j i}\right)=\frac{\mu_{i j}-v_{i j}+1}{2}+\frac{\mu_{j i}-v_{j i}+1}{2}$.

Because $B=\left(b_{i j}\right)$ is an IFPR then $\mu_{i j}=v_{j i}$ and $v_{i j}=\mu_{j i}$, and therefore it is true that

$$
p_{i j}+p_{j i}=1 \forall i, j \in\{1, \ldots, n\} .
$$

The following result proves that this method is consistent with the case when the IFPR reduces to be a FPR, in which case the SFPR coincides with the original FPR.

Corollary 1. Let $B=\left(b_{i j}\right)$ be an IFPR and $P=\left(p_{i j}\right)$ its associated SFPR. If the hesitancy degree function is the null function then $B=P$.

C. Fuzzy Quantifier Guided Selection Approaches based on Dominance and Non-dominance Degree for Fuzzy Sets

Yager presented in [30] a methodology to formulate linguistic expressions using ordered weighted average (OWA) operators guided by fuzzy linguistic quantifiers [32].

A fuzzy linguistic quantifier is modelled by means of basic unit-monotonic (BUM) function, $Q$, i.e.

$$
\begin{gathered}
Q:[0,1] \rightarrow[0,1] \\
Q(0)=0 \\
Q(1)=1 \\
Q(x) \geq Q(y) \text { if } x \geq y .
\end{gathered}
$$

The OWA operator weights are calculated as follows:

$$
w_{i}=Q\left(\frac{i}{n}\right)-Q\left(\frac{i-1}{n}\right), i=1, \ldots, n .
$$

These non-decreasing relative fuzzy linguistic quantifiers have been modelled in the literature with the following BUM function $Q$

$$
Q(x)=\left\{\begin{array}{cc}
0 & 0 \leq x<a \\
\frac{x-a}{b-a} & a \leq x \leq b \\
1 & b<x \leq 1
\end{array}\right.
$$

$a, b \in[0,1]$. Notice that the election of different values for the parameters $a$ and $b$ could lead to different types of fuzzy quantifiers, for instance the fuzzy linguistic quantifier 'most of' has been usually implemented by choosing the values $(a, b)=(0.3,0.8)$. Other membership functions are possible though, and the parameterised family of regular increasing monotone (RIM) quantifiers $Q(r)=r^{a}(a \geq 0)$ suggested for by Yager in [29] is one of them. This family of functions guarantees that: (i) all the experts contribute to the final aggregated value (strict monotonicity property), and (ii) associates, when $a \in[0,1]$, higher weight values to the aggregated values with associated higher importance values (concavity property) [10]. In particular, the value $a=1 / 2$ is suggested to be used to represent the fuzzy linguistic quantifier 'most of'.

Based on this methodology, a fuzzy quantifier guided nondominance degree, which extends Orlovsky's non-dominance concept, for a FPR was presented in [8].

Given a fuzzy preference relation $R=\left(r_{i j}\right) \mid \forall i j: r_{i j} \in[0,1]$ :

1) The Fuzzy Quantifier Guided Dominance Degree $(Q G D D)$ for the alternative $x_{i}$ quantifies the dominance that alternative $x_{i}$ has over the fuzzy majority of the remaining alternatives:

$$
Q G D D_{i}=\phi_{Q}\left(r_{i j}, j=1, \ldots, n\right),
$$

with $\phi_{Q}$ being an OWA operator guided by the fuzzy linguistic quantifier represented by the BUM function $Q$.

2) The Fuzzy Quantifier Guided Non Dominance Degree $(Q G N D D)$ for the alternative $x_{i}$ quantifies the degree up to which such alternative is not dominated by a fuzzy majority of the remaining alternatives:

$$
Q G N D D_{i}=\phi_{Q}\left(1-r_{j i}^{s}, j=1, \ldots, n\right),
$$

with $r_{j i}^{s}=\max \left\{r_{j i}-r_{i j}, 0\right\}$ representing the degree up to which $x_{i}$ is strictly dominated by $x_{j}$ [9].

\section{Comparative Analysis of the Ranking APPROACHES FOR IFPR AND FPR}

The score degree plays a key role in multi-attribute decision making problems where the experts' preferences are expressed by means of intuitionistic fuzzy information since they allow the comparison of an alternative with respect to a criterion or with respect to another alternative using intuitionistic fuzzy sets. In the same way, dominance and non-dominance based approaches are relevant to obtain a ranking of the alternatives when the expert's preferences are given by means of fuzzy preference relations. In the previous section we have demonstrated the existence of a one-to-one relationship between the set of IFPS and the subset of the sort of FPRs that are asymmetric. With this in mind, in this section we analyse the relation between the main ranking approaches proposed for both types of preference relations.

\section{A. IFPR Score and Accuracy Degrees in Terms of Asymmetric $F P R$}

In the following we analyse the relation between the score and accuracy functions defined for the case of intuitionistic fuzzy preference relations and the asymmetric fuzzy preference relations, taking into consideration the two score degrees presented in the previous section. 
Theorem 1. Let $B=\left(b_{i j}\right)$ with $b_{i j}=\left\langle\mu_{i j}, v_{i j}\right\rangle$ be an IFPR with equivalent asymmetric fuzzy preference relation $R=\left(r_{i j}\right)$. Then:

- Chen and Tan's score value, $S_{C T}(B)$, can be represented as follows:

$$
S_{C T}\left(b_{i j}\right)=r_{i j}-r_{j i} \quad \forall i j
$$

- Hong and Choi's accuracy value, $A_{H C}(B)$, is expressed as follows:

$$
A_{H C}\left(b_{i j}\right)=r_{i j}+r_{j i} \quad \forall i j
$$

Proof. According to the one-to-one correspondence between the set of intuitionistic fuzzy preference relations and the set of asymmetric reciprocal fuzzy preference relations $r_{i j}=\mu_{i j}$ and under reciprocity $\left\langle\mu_{i j}, v_{i j}\right\rangle=\left\langle\mu_{j i}, v_{j i}>\right.$ and so $v_{i j}=\mu_{j i}$ and $v_{i j}=r_{j i}$.

\section{B. Non-dominance Degree and Score Degree Relationships}

In the following the analysis of the relationships between the non-dominance degrees used to rank the fuzzy preference relations and the score degrees for the case of intuitionistic fuzzy preference relations is carried out.

1) Relationship between the Non-dominance Degree and Cheng and Tan's Score Degree.

Theorem 2 (Non-dominance via Cheng and Tan's score function). Let $B=\left(b_{i j}\right)$ with $b_{i j}=\left\langle\mu_{i j}, v_{i j}\right\rangle$ be an IFPR with equivalent asymmetric fuzzy preference relation $R=$ $\left(r_{i j}\right)$. The non-dominance degree associated to $R$ can be represented in terms of the Chen and Tan's score value, $S_{C T}$, associated to $B$, as follows:

$$
Q G N D D_{i}=\phi_{Q}\left(1+\min \left\{S_{C T}\left(b_{i j}\right), 0\right\}, \quad j=1, \ldots, n\right),
$$

Proof. Recall that

$$
Q G N D D_{i}=\phi_{Q}\left(1-r_{j i}^{s}, j=1, \ldots, n\right),
$$

with

$$
r_{j i}^{s}=\max \left\{r_{j i}-r_{i j}, 0\right\}
$$

Taking into consideration the one-to-one correspondence between the set of intuitionistic fuzzy preference relations and the set of asymmetric fuzzy preference relations, $r_{j i}^{s}$ can be expressed by means of the membership and non membership values in a intuitionistic fuzzy preference relation as follows:

$$
r_{j i}^{s}=\max \left\{v_{j i}-\mu_{i j}, 0\right\}
$$

Given that

$$
S_{C T}\left(b_{i j}\right)=\mu_{i j}-v_{i j},
$$

the degree up to which $x_{i}$ is strictly dominated by $x_{j}$ can be expressed in terms of the score value $S_{C T}$ as follows:

$$
r_{j i}^{s}=\max \left\{-S_{C T}\left(b_{i j}\right), 0\right\}
$$

Under reciprocity $-S_{C T}\left(b_{i j}\right)=S_{C T}\left(b_{j i}\right)$. Therefore

$$
\begin{aligned}
1-r_{j i}^{s} & =1-\max \left\{S_{C T}\left(b_{j i}\right), 0\right\}=\min \left\{1-S_{C T}\left(b_{j i}\right), 1\right\} \\
& =\min \left\{1+S_{C T}\left(b_{i j}\right), 1\right\}=1+\min \left\{S_{C T}\left(b_{i j}\right), 0\right\}
\end{aligned}
$$

2) Relationship between the Non-dominance Degree and $\mathrm{Wu}$ and Chiclana's Score Degree.

Theorem 3 (Non dominance via $\mathrm{Wu}$ and Chiclana's score FPR associated to an IFPR). Let $B=\left(b_{i j}\right)$ with $b_{i j}=\left\langle\mu_{i j}, v_{i j}\right\rangle$ be an IFPR with equivalent asymmetric fuzzy preference relation $R=\left(r_{i j}\right)$. The fuzzy quantifier guided non-dominance degree associated to $R$ can be represented in terms of Wu and Chiclana's score value, $S_{W C}$, associated to $B$, as follows:

$$
\left.Q G N D D_{i}=\phi_{Q}\left(1-S_{W C}^{S}\left(b_{j i}\right), 0\right\}, \quad j=1, \ldots, n\right),
$$

with $S_{W C}^{s}\left(b_{j i}\right)=\max \left\{S_{W C}\left(b_{j i}\right)-S_{W C}\left(b_{i j}\right), 0\right\}$

Proof. The following holds:

$$
\begin{aligned}
S_{W C}\left(b_{i j}\right)-S_{W C}\left(b_{j i}\right) & =\frac{\mu_{i j}-v_{i j}+1}{2}-\frac{\mu_{j i}-v_{j i}+1}{2} \\
& =\frac{\left(\mu_{i j}+v_{j i}\right)-\left(\mu_{j i}+v_{i j}\right)}{2}
\end{aligned}
$$

Because $B=\left(b_{i j}\right)$ is an IFPR then $\mu_{i j}=v_{j i}$ and $v_{i j}=\mu_{j i}$, and therefore it is

$$
S_{W C}\left(b_{i j}\right)-S_{W C}\left(b_{j i}\right)=\frac{2 \mu_{i j}-2 \mu_{j i}}{2}=\mu_{i j}-\mu_{j i}
$$

Taking that $\mu_{i j}=r_{i j}$, it is

$$
r_{j i}^{s}=\max \left\{r_{j i}-r_{i j}, 0\right\}=\max \left\{S_{W C}\left(b_{j i}\right)-S_{W C}\left(b_{i j}\right), 0\right\}
$$

Summarising

$$
r_{j i}^{s}=S_{W C}^{s}\left(b_{j i}\right)
$$

\section{Fuzzy Non-dominance Degree for Intuitionistic Fuzzy Pref-} erence Relations

Let $B=\left(b_{i j}\right)$ with $b_{i j}=\left\langle\mu_{i j}, v_{i j}\right\rangle$ be an IFPR. It has been proved that two FPRs can be associated to the IFPR:

- The asymmetric FPR: $R=\left(r_{i j}\right)=\left(\mu_{i j}\right)$.

- The score FPR: $P=\left(p_{i j}\right)=\left(S_{W C}\left(b_{i j}\right)\right)$.

Notice that in preference modelling, given an asymmetric FPR, it is always possible to derive a reciprocal FPR. When this procedure is applied, $P$ is the reciprocal FPR that derives from $R$.

A procedure to rank alternatives assessed via an IFPR $B$ could therefore be performed by applying the fuzzy quantifier guided non-dominance degree to one of its two associated FPRs given above. The issue that arises here is that the ranking of alternatives could be different depending on which FPR is used, i.e. the fuzzy quantifier guided non-dominance degree obtained via FPR $R$ could be different to the fuzzy quantifier non-dominance degree obtained via FPR $P$ and ultimately could result in different ranking of alternatives. 
This, obviously, is an undesirable outcome that does not happen as Theorem 3 proved.

Indeed, let us denote by $I Q G N D D^{I}$ and $I Q G N D D^{S}$ the fuzzy quantifier guided non-dominance degree of an IFPR computed via its equivalent asymmetric FPR and score FPR, respectively, i.e.

$$
\begin{aligned}
I Q G N D D_{i}^{I} & =\phi_{Q}\left(1-r_{j i}^{s}, j=1, \ldots, n\right) \\
I Q G N D D_{i}^{S} & =\phi_{Q}\left(1-p_{j i}^{s}, j=1, \ldots, n\right)
\end{aligned}
$$

with $r_{j i}^{s}=\max \left\{r_{j i}-r_{i j}, 0\right\}$ and $p_{j i}^{s}=\max \left\{p_{j i}-p_{i j}, 0\right\}$.

In Theorem 3, it was proved that

$$
r_{j i}^{s}=\max \left\{r_{j i}-r_{i j}, 0\right\}=\max \left\{S_{W C}\left(b_{j i}\right)-S_{W C}\left(b_{i j}\right), 0\right\}
$$

and therefore it is

$$
p_{j i}^{s}=r_{j i}^{s}
$$

Consequently,

$$
I Q G N D D_{i}^{I} \equiv I Q G N D D_{i}^{S}
$$

This result motivates the following definition of the fuzzy quantifier guided non-dominance degree of an IFPR.

Definition 9. Let $B=\left(b_{i j}\right)$ with $b_{i j}=\left\langle\mu_{i j}, v_{i j}\right\rangle$ be an IFPR. The fuzzy quantifier guided non-dominance degree of alternative $x_{i}$ measures the degree up to which such alternative is not dominated by a fuzzy majority of the remaining alternatives, and it is expressed as follows:

$$
I Q G N D D_{i}=\phi_{Q}\left(1-\mu_{j i}^{s}, j=1, \ldots, n\right),
$$

with $\mu_{j i}^{s}=\max \left\{\mu_{j i}-\mu_{i j}, 0\right\}$.

The fuzzy quantifier guided dominance degree when applied to the equivalent asymmetric FPR, $R$, is different to the fuzzy quantifier guided dominance degree obtained when applied to the score FPR, $P$. These only coincide when hesitancy degree is the null function, i.e, $\tau \equiv 0$. As dominance is based on the level of degree of preference of an alternative over another one, it makes sense in the case of an IFPR to define its corresponding fuzzy quantifier guided dominance degree as that of its equivalent asymmetric FPR.

Definition 10. Let $B=\left(b_{i j}\right)$ with $b_{i j}=\left\langle\mu_{i j}, v_{i j}\right\rangle$ be an IFPR. The fuzzy quantifier guided dominance degree of alternative $x_{i}$ measures the degree up to which such alternative dominates a fuzzy majority of the remaining alternatives, and it is expressed as follows:

$$
\begin{gathered}
I Q G D D_{i}=\phi_{Q}\left(\mu_{i j}, j=1, \ldots, n\right) . \\
\text { V. CONCLUSIONS }
\end{gathered}
$$

Ranking fuzzy numbers is indispensable in group decision making methodologies so as to obtain the best alternative from the experts' opinions. In this contribution we have compared the methodologies proposed for intuitionistic fuzzy preference relations (based on score and accuracy functions) and for fuzzy preference relations (based on dominance and non dominance degrees).
This study has been based on the proved one-to-one correspondence between the intuitionistic fuzzy preference relations and asymmetric fuzzy preference relations [22]. It has also covered previous proposal to derive a reciprocal fuzzy preference relation from an intuitionistic fuzzy preference relation via the application of score function provided in in [24]. It has been proved that the fuzzy quantifier guided non-dominance degree of both the asymmetric fuzzy preference relation and score fuzzy preference relation associated to an intuitionistic fuzzy preference relation coincide.

The significance of this result resides in that in practice it allows for the introduction of the fuzzy quantifier guided nondominance degree concept for intuitionistic fuzzy preference relations in an unique and appropriate way.

\section{ACKNOWLEDGMENTS}

The authors would like to acknowledge the support from FEDER funds in the FUZZYLING-II Project TIN2010-17876, as well as the support from the Andalusian Excellence Projects TIC-05299 and TIC-5991.

\section{REFERENCES}

[1] S. Alonso, F. Cabrerizo, F. Chiclana, F Herrera, and E. Herrera-Viedma. Group decision making with incomplete fuzzy linguistic preference relations. Int. J. Intell. Syst, 24(2):201-222, 2009.

[2] K. T. Atanassov. Intuitionistic fuzzy sets. Fuzzy Sets and Systems, 20(1):87-96, 1986.

[3] J.C. Bezdek, B. Spillman, and R. Spillman R. A fuzzy relation space for group decision theory. Fuzzy Sets and Systems, 1(4):255-268, 1978.

[4] M. Brunelli, and J. Mezei. How different are ranking methods for fuzzy numbers? A numerical study. International Journal of Approximate Reasoning, 54 (5), 627-639, 2013.

[5] H. Bustince and P. Burillo. Vague sets are intuitionistic fuzzy sets. Fuzzy Sets and Systems, 79(3):403 - 405, 1996.

[6] S.-M. Chen and J.-M. Tan. Handling multicriteria fuzzy decision-making problems based on vague set theory. Fuzzy Sets and Systems, 67(2):163172, 1994.

[7] Ting-Yu Chen. A comparative analysis of score functions for multiple criteria decision making in intuitionistic fuzzy settings. Information Sciences, 181(17):3652 - 3676, 2011.

[8] F. Chiclana, F. Herrera, and E. Herrera-Viedma. Integrating three representation models in fuzzy multipurpose decision making based on fuzzy preference relations. Fuzzy Sets and Systems, 97(1):33 - 48, 1998.

[9] F. Chiclana, F. Herrera, and E. Herrera-Viedma. A note on the internal consistency of various preference representations. Fuzzy Sets and Systems, 131(1):75 - 78, 2002.

[10] F. Chiclana, E. Herrera-Viedma, F. Herrera, and S. Alonso. Some induced ordered weighted averaging operators and their use for solving group decision-making problems based on fuzzy preference relations. European Journal of Operational Research, 182(1):383-399, 2007.

[11] P.C. Fishburn. Utility theory for decision making. Krieger, Melbourne, FL, 1979

[12] Hamido Fujita, Masaki Kurematsu, and Jun Hakura. Virtual doctor system (vds) and ontology based reasoning for medical diagnosis. In Endre Pap, editor, Intelligent Systems: Models and Applications, volume 3 of Topics in Intelligent Engineering and Informatics, pages 197-214. Springer Berlin Heidelberg, 2013.

[13] W.-L. Gau and D.J. Buehrer. Vague sets. Systems, Man and Cybernetics, IEEE Transactions on, 23(2):610-614, Mar 1993.

[14] F. Herrera and E. Herrera-Viedma. Choice functions and mechanisms for linguistic preference relations. European Journal of Operational Research, 120(1):147-161, 2000.

[15] E. Herrera-Viedma, F. Chiclana, F.Herrera, and S. Alonso. Group decision-making model with incomplete fuzzy preference relations based on additive consistency. IEEE Transactions on Systems, Man, and Cybernetics, Part B: Cybernetics, 37(1):176-189, 2007. 
[16] Dug Hun Hong and Chang-Hwan Choi. Multicriteria fuzzy decisionmaking problems based on vague set theory. Fuzzy Sets and Systems, 114(1): 103 - 113, 2000.

[17] I. Millet. The effectiveness of alternative preference elicitation methods in the analytic hierarchy process. Journal of Multi-Criteria Decision Analysis, 6(1):41-51, 1997.

[18] S.A. Orlovsky. Decision-making with a fuzzy preference relation. Fuzzy Sets and Systems, 1(3):155 - 167, 1978.

[19] P. Pérez-Asurmendi and F. Chiclana. Linguistic majorities with difference in support. Applied Soft Computing, 18:32-39, 2014.

[20] M. Roubens and P. Vincke. Preference modeling. Springer, Berlin, 1985.

[21] E.E. Szmidt and J. Kacprzyk. Using intuitionistic fuzzy sets in group decision making. Control and Cybernetics, 31(4):1037-1053, 2002.

[22] Raquel Urena, Francisco Chiclana, Hamido Fujita, and Enrique HerreraViedma. Confidence-consistency driven group decision making approach with incomplete reciprocal intuitionistic preference relations. Knowledge-Based Systems, 89:86 - 96, 2015.

[23] Ioannis K. Vlachos and George D. Sergiadis. Intuitionistic fuzzy information - applications to pattern recognition. Pattern Recognition Letters, 28(2):197 - 206, 2007.

[24] J. Wu and F. Chiclana. Non-dominance and attitudinal prioritisation methods for intuitionistic and interval-valued intuitionistic fuzzy preference relations. Expert Systems with Applications, 39(18):13409-13416, 2012.
[25] Jian Wu and Francisco Chiclana. Multiplicative consistency of intuitionistic reciprocal preference relations and its application to missing values estimation and consensus building. Knowledge-Based Systems, 71(0): 187 - 200, 2014

[26] Z. Xu, X. Cai, and E. Szmidt. Algorithms for estimating missing elements of incomplete intuitionistic preference relations. International Journal of Intelligent Systems, 26(9):787-813, 2011.

[27] Z. S. Xu. A survey of preference relations. International Journal of General System, 27(36):179-203, 2007.

[28] Zeshui Xu, Jian Chen, and Junjie Wu. Clustering algorithm for intuitionistic fuzzy sets. Information Sciences, 178(19):3775 - 3790, 2008.

[29] R.R. Yager. Quantifier guided aggregation using OWA operators. International Journal of Intelligent Systems, 11(1):49-73, 1996.

[30] R. R. Yager. Owa aggregation over a continuous interval argument with applications to decision making. IEEE Transactions on Systems, Man, and Cybernetics-Part B: Cybernetic, 34:1952-1963, 2004.

[31] L. A. Zadeh. Outline of a new approach to the analysis of complex systems and decision processes. IEEE Transactions on Systems, Man and Cybernetics, 3:28-44, 1973.

[32] Lotfi A. Zadeh. A computational approach to fuzzy quantifiers in natural languages. Computers and Mathematics with Applications, 9(1):149 184, 1983. 